\title{
Pantalla o papel. La construcción lectora en contexto digital y analógico en hablantes de lenguas próximas
}

ANTONIO CHENOLL

Universidade Católica Portuguesa (CECC)

A_chenoll@fch.lisboa.ucp.pt

\begin{abstract}
Resumen: En este trabajo se pretende delinear la importancia de conocer explícitamente los condicionantes del proceso de lectura desde una perspectiva contextual (digital y analógica) para una optimización del proceso de aprendizaje en hablantes de lenguas afines. Para ello, se diseñó una prueba cuantitativa mediante la cual poder medir de manera más objetiva posible la capacidad de construcción de un cerebro lingüístico cuya lengua materna posee afinidades tan marcadas como es el portugués en relación con el español. Realizar un punto de comparación entre ambos grupos nos ayudó a conocer el nivel en el que los alumnos usan de una manera más o menos completa el sesgo informativo como elemento de ahorro cognitivo. Los resultados de esta comparación nos ayudan a afirmar que existe una diferencia en ambos contextos y recomendar, según en qué situaciones, usar un formato $\mathrm{u}$ otro dependiendo de nuestras necesidades como docentes o como discentes.
\end{abstract}

Palabras clave: Percepción, E/LE, Lenguas afines, digital, lectura.

Screen or paper. Reading construction in digital and analogical context in speakers of nearby languages

\begin{abstract}
This paper aims to delineate the importance of knowing explicitly the conditioning factors of the reading process from a contextual perspective (digital and analog) for an optimization of the learning process in speakers of related languages. For this, a quantitative test was designed to measure in a more objective way the construction capacity of a linguistic brain whose mother tongue has such marked affinities as Portuguese in relation to Spanish. Making a point of comparison between both groups helped us to know the level at which students use in a more or less complete way the information bias as an element of cognitive saving. The results of this comparison help us to affirm that there is a difference in both contexts and recommending, according to which situations, to use one format or another depending on our needs as teachers or as learners.
\end{abstract}

Key words: Perception, S/FL, Related Languages, digital, reading.

\section{Introducción}

Parece innegable y a veces incluso demasiado evidente que muchos de los contextos sociales y laborales deben pasar inevitablemente por la vía digital. El proceso de enseñanza y aprendizaje no es, ni puede ser una excepción. Esta vía no es solo una fuente de información a la que acuden una parte muy significativa de nuestros alumnos y nosotros mismos, como también es el canal en el que se produce el conocimiento actual 
de una o de otra manera. En este sentido, la tendencia a la lectura en formato digital parece ser una vía a la que debemos prestar atención no solo desde el punto de vista de la veracidad de la información, sino también desde el punto de vista del proceso cognitivo de aprendizaje.

De especial importancia se reviste este hecho cuando somos conscientes de la realidad de nuestros alumnos, como usuarios de una percepción de la realidad constructivista, los cuales utilizan los llamados sesgos cognitivos de confirmación de la información con el objetivo inconsciente de ahorrar carga cognitiva (McMillan y Review 1993). Esto es, ante determinada situación que exige una relativamente alta carga cognitiva, rellenan información a partir de las expectativas creadas. Como veremos más adelante, estas expectativas aumentan significativamente cuando nos enfrentamos a hablantes de lengua próximas o afines.

Si a la ecuación en la que, de momento, intervienen (1) elementos de lengua afín y (2) construcción de realidad lingüística; le sumamos el elemento de la lectura en formato digital, obtendremos un interesante resultado que debe ser estudiado tanto por sus partes individuales como por la suma de estas de manera que obtengamos un marco en el que realmente seamos conscientes de cuáles son los elementos que debemos tener en cuenta en el proceso de lectura de este perfil específico.

La construcción de la lectura digital en hablantes de lenguas afines será, pues, un condicionante imprescindible no solo en la metodología aplicada a este tipo de perfiles cognitivos; sino en el diseño de materiales e, incluso, en el propio proceso de autonomía.

Los aprendientes, a su vez, como sujetos creativos de la realidad deben ser conscientes de que, en los primeros estadios de aprendizaje explícito, la lectura de una lengua afín debe realizarse de manera pausada y analítica que ayude al verdadero objetivo del aprendizaje formal: la optimización del proceso. Esto es, conseguir en menos tiempo mejores y más duraderos resultados.

Es en este sentido que proponemos un análisis basado en los errores producidos por hablantes de lenguas afines mediante los cuales nos acercamos o intuimos a los procesos cognitivos que subyacen en el perfil descrito. Para ello, haremos un análisis cuantitativo de los datos que ayuden a interpretar de manera objetiva y alejen tanto cuando sea posible la subjetividad del observador.

Finalmente, la pregunta que cabe realizarse en este trabajo es si de entre los diferentes contextos visuales en los que el alumno pone en evidencia sus errores, puede existir una diferencia cuando una lectura se realiza en un ambiente digital o en un ambiente analógico.

\section{Marco teórico}

\subsection{Cómo lee el cerebro de una lengua afín}

Como sabemos, el proceso de lectura en una lengua materna no es un proceso continuo de la misma manera en como leen los niños o las personas semianalfabetas en su lengua materna. Cuando aprendemos a conducir, pensamos explícitamente en todos los movimientos que estamos realizando; cuando aprendemos a leer, la lectura es excesivamente analítica y nada funcional (Bravo Valdivieso 2016; Piquer 2016). 
Conforme aprendemos a mecanizar los movimientos aprendemos realmente a conducir con soltura y podemos realizar otras actividades mínimas sin mayor esfuerzo. De manera análoga, cuando aprendemos a leer tendemos a mecanizar el proceso que se sustituye por un proceso de adelantamiento en función de las expectativas sumadas a pistas físicas y metalingüísticas que son, en este caso concreto, las letras de la lectura y lo esperable lingüísticamente. Este atajo cognitivo nos ayuda a optimizar el proceso de lectura y por lo tanto a ser más eficaces y rápido cuando leemos. Cuando somos lectores expertos, aprendemos a analizar el texto de manera esquemática con el objetivo de liberar esa carga cognitiva que sería el leer letra a letra todo el texto. Ejemplo de ello es el conocido texto en el que las letras están desordenadas en su interior manteniendo únicamente el inicio y el final de cada palabra y aun así somos capaces de leerlo sin mayores problemas. De la misma manera, sabemos a través del seguimiento ocular (eyes tracking) que la lectura se realiza de una manera no lineal en el que el lugar en el que los ojos centran su atención no es una secuenciación de principio-fin, sino más bien de principio-fin-principio.

Por lo tanto, es obvio que, en el proceso de lectura de manera analítica, exista un relleno de información en el que el cerebro realiza un trabajo de predicción (Hohwy 2013; Ransom, Fazelpour, y Mole 2017). Es decir, rellena una información explícita que supone por diferentes motivos. Por un lado, por la referencia gráfica (letras), por otro lado, la expectavilidad y de valencias posibles en una cadena determinada. A modo de ejemplo, si leemos una frase como «La casa de mi madre está muy lejos.» existe el proceso de descodificación analítica en la que podemos leer antes «casa» que «la», ya que los artículos no aportan información relevante en la mayor parte de los casos. Por otro lado, podemos leer «está» y pensar implícitamente que «lejos» es una posible valencia ante «muy». De esta manera no es necesario leer toda la frase palabra por palabra. Es un proceso que se relaciona con la minimización del error y el ahorro de carga cognitiva de hecho lingüístico de comprensión.

Desde el punto de vista de la lectura en una lengua extranjera afín, el proceso de lectura funciona de manera muy similar al de un hablante nativo. Leemos, construimos realidad dependiendo de las referencias mínimas que escogemos inconscientemente de nuestras expectativas y valoramos las diferentes valencias. El problema reside en que las valencias y nuestras expectativas, así como los diferentes referentes mínimos que escogemos nos puede llevar a leer algo que no está escrito realmente. Es decir, cuando un hablante de una lengua próxima o afín al español lee un texto en la lengua meta, utiliza las mismas herramientas cognitivas para minimizar el impacto de la carga cognitiva. No obstante, en su proceso de lectura, el hablante de una lengua afín a diferencia del hablante experto (nativo), tiende a definir las expectativas según su lengua materna o según otras lenguas afines en la que haya desarrollado una interlengua ${ }^{1}$ (Fernández 1997; Nemser 1992; Tarone 1994).

\footnotetext{
${ }^{1}$ Debe entenderse la interlengua como el sistema lingüístico no-nativo de una aprendiente. Es decir, la lengua idiosincrásica de cada aprendiente en la lengua extranjera que está adquiriendo. Este concepto surge con Selinker $(1969,1972)$ y ha sido usado por varios autores que lo denominaron como «dialecto idiosincrásico» (Corder,1971) o «sistema aproximado» (Nemser, 1971) entre otras. Lo que resulta interesante en esta idea es la imagen mental sobre una lengua interna interconectada a la lengua materna, en evolución y que depende en gran medida del diálogo entre la lengua meta y la construcción personal del alumno.
} 
Tal como los hablantes de una lengua nativa realizan sus expectativas teniendo en cuenta la situación contextual y lingüística en la que se encuentra, los no-nativos lo realizan fuertemente influenciados por su interlengua. Por lo tanto, parece evidente plantear como hipótesis que el nivel de creación de realidad lingüística en la lectura de un texto será directamente proporcional al estadio alcanzado en su interlengua. Evidentemente, esta hipótesis debe ser considerada como un todo. Es decir, si el hablante no-nativo desconoce cierta palabra en la lengua meta, tenderá a leer esa misma palabra según la lengua materna si se dan algunos condicionante como hemos visto anteriormente. Estos condicionantes pueden describirse como:

a. La palabra o estructura es lo suficientemente parecida con una palabra similar en otra interlengua del hablante.

b. La palabra contiene elementos gráficos similares en la interlengua.

c. Las expectativas nos guían la estructura siguiente.

d. Las posibles valencias son pocas en la lengua materna.

Teniendo en cuenta estos factores, podremos intuir que la interlengua y la expectación tendrán una gran influencia en el proceso de lectura. Esta influencia se ve fuertemente condicionada en el caso de aprendientes con una L1 afín o con L2 afines.

\subsection{Importancia del proceso de lectura en el aprendizaje de una lengua extranjera y próxima}

La lectura en una lengua extranjera es uno de los ejercicios que pueden resultar más efectivos cuando pretendemos mejorar nuestras competencias activas y pasivas de la lengua. La diferencia fundamental con otro tipo de ejercicios es que podemos analizar las muestras de lengua con la suficiente antelación para percibir las pequeñas diferencias que puedan existir con la lengua materna del alumno, o bien, descubrir errores de nuestra propia interlengua. Este fenómeno tiene sus raíces en la propia idiosincrasia del proceso de lectura que nos permite observar el referente escrito con el fonético y nos permite parar el continuun de lectura para revisar el texto. Al contrario, en el proceso de escucha natural la carga cognitiva privilegia el componente semántico al componente formal en, por ejemplo, una conversación. A pesar de que esta ventaja no sea exclusiva del proceso de lectura (es posible parar una grabación, aunque no es posible parar una conversación para volver atrás sin costes comunicativos), es mucho más probable hacerlo en el proceso de lectura.

No obstante, de la misma manera que sucede en el proceso de escucha activa, en el proceso de lectura se evidencia la tendencia de predictibilidad con la que se rebaja la carga cognitiva del proceso de descodificación e interpretación de una lengua extranjera. De la misma manera que sucede con la escucha, la mente predictiva (Hohwy 2013; Ransom et al. 2017) realiza predicciones sobre qué es lo que va a suceder a continuación. Este concepto aplicado al proceso de inferencia se puede resumir en que cuando la interlengua de un hablante no nativo considera que una palabra se escribe de determinada forma, existe un alto índice de percibirla de esa manera y no tal y como está escrita.

Las ventajas de este proceso son innumerables. Gracias a esta técnica podemos entender otras lenguas próximas, entender las variantes de una misma lengua o reconocer 
elementos como siendo de una única categoría. De la misma manera, la carga cognitiva limitada de nuestro cerebro se concentra en aquellas acciones que en ese momento nos resulten más atractivas para nuestro objetivo comunicativo.

Pero ¿cómo podemos aprovechar este error natural de una mente sana?

Acceder a la interlengua del alumno es, de momento, una tarea a la que solo nos podemos aproximar de manera contaminada por los elementos como las producciones escritas y orales, pero siempre corremos el riesgo de que la prueba pueda ser contaminada por diferentes factores contextuales que, aunque no inviabilizan completamente la muestra obtenida, sí que gradualmente se ven influenciadas por los condicionantes propios de los actos de habla. Algunos ejemplos como el nerviosismo, el nivel de confianza con el interlocutor, la exigencia de la rapidez de la respuesta, entre otros, es buena prueba de ello. No obstante, la producción es una manera de inferir el estadio de la interlengua en la que se encuentran los hablantes de una lengua extranjera. Sin embargo, de la misma manera que no podemos entrar en la mente de una persona, solo podemos aproximarnos a ella a través de elementos mediante los cuales inferimos las características internas. Esto es, podemos expresar nuestro pensamiento a través de signos lingüísticos que previamente han pasado por un filtro que, como hemos dicho, desconfigura en mayor o menor grado el mensaje. En definitiva, podemos afirmar que el error es un explicitador de la interlengua del alumno. Esto es, a través de los errores podemos ver qué pasa dentro del hablante de una lengua extranjera, podemos inferir cuáles son los procesos que subyacen a la producción de la misma manera que a través de un error en un sistema informático podemos deducir cuáles son los errores de programación que están resultando dañinos para el normal funcionamiento de la máquina.

En esta línea de pensamiento, aunque sabemos que el error es un explicitador de la interlengua (Fernández 1997), así como un marcador del nivel de lengua, no conviene confundir los conceptos propuestos en Tarone en el que diferencia entre falta (mistake) y error (error). El error se considera un distanciamiento sistemático de la regla de la lengua meta, mientras que la falta es un error exclusivo de ese momento dado por condicionamientos que no se pueden achacar a que el alumno no sea consciente de esta etiqueta de error. En otras palabras, el error es sistemático, mientras que la falta es esporádica. La importancia de esta diferenciación reside en su clasificación no solo como parte integrante de la interlengua desviada, como porque exige el cuidado requerido por parte del aprendiente y del docente para que no se transforme en una fosilización. No obstante, desde el punto de vista del análisis de la interlengua que nos ocupa, tanto el error como la falta, serán los elementos que nos aporten una información relevante.

En otros trabajos, hemos demostrado cómo la lectura de un texto auditivo evidencia errores marcados por la influencia de la interlengua (Chenoll 2016a; 2016b). Cabe aquí preguntarnos si esas evidencias están también relacionadas con la lectura en el contexto digital/analógico en el que se producen.

\subsection{Diferencias entre la lectura en papel y en formato digital}

Así, debemos cuestionarnos sobre las características de cada formato y las posibles influencias que pueden repercutir en la prueba de lectura. 
Como veremos más adelante, la prueba fue realizada en dos formatos diferentes. Por un lado, se obtuvo datos de informantes que leyeron el texto a través de una tableta digital y por otros informantes que realizaron la prueba en formato analógico.

Desde el punto de vista cognitivo y del procesamiento de la información analítico, podemos observar que la máxima diferencia y a la que deberemos prestar atención se refiere al origen del foco de iluminación en ambos formatos. Si desde el punto de vista de un papel, la luz con la que se lee es un reflejo indirecto de otros focos de luz, en el caso de la tableta, la luz proviene directamente del dispositivo. Esto puede llegar a afectar los procesos de discriminación de las palabras en el momento de la lectura.

Otras características más prácticas como el hecho de poder tener acceso a varios textos, el aumento del tipo de letra, así como la manejabilidad del aparato, fueron debidamente neutralizados ya que se intentó que, mediante las definiciones del dispositivo fueran lo más parecidos posible tanto de tamaño como de luminosidad o tipo de letra utilizada.

Por lo tanto, consideramos que se consiguió aislar el problema para que solo el proceso de lectura influenciara sobre el resultado. Es importante señalar que nos interesa el proceso de predicción y de creación de realidad, procesos estos cognitivos que queremos aislar de elementos externos de un contexto online.

\section{Metodología}

Con el objetivo citado de aislar el problema fue diseñada una prueba en la que los informantes se expondrían a un texto escrito en un formato digital a través de una tableta para el grupo experimental y a un texto en formato analógico para el grupo de control.

Para la realización de la prueba se tuvo en consideración el aislamiento de los elementos que pudieran ser considerados como no relevantes en los procesos cognitivos de lectura y que pudiera tener una influencia en los resultados de estos. De la misma manera, para la validación y correcta comparación de los resultados, fueron utilizados únicamente los informantes de lengua portuguesa ya que de esta manera existiría una estandarización de los datos recogidos.

Se consideró que la mejor manera de comparar los datos sería desde una perspectiva cuantitativa ya que el texto puede ser aislado por elementos divisibles. Así, se consigue que exista una comparación para determinar objetivamente el porcentaje de errores y aciertos de modo objetivo y comparable.

Finalmente, se consideró fundamental la grabación de la prueba para un posterior análisis sin elementos de distracciones para el observador que pudiesen desvirtualizar el objetivo de esta.

\subsection{Descripción de la prueba}

La prueba consistió en la lectura de un texto en el que se incluyen elementos que puedan suponer un problema de lectura en hablantes lusófonos con el objetivo de observar el componente de predicción según el nivel de interlengua de los alumnos. No obstante, como método de comparación el grupo de control leyó el texto de manera analógica, mientras que el grupo experimental lo hizo a través de una tableta. 
Cabe mencionar que los informantes no conocen la finalidad del ejercicio, aunque saben que están siendo grabados con fines experimentales.

En lo que se refiere al universo de los informantes se seleccionó a aquellos que tenían un perfil común con el objetivo de que las pruebas pudieran ser falseables y comparables.

El perfil de los informantes se corresponde a 68 alumnos lusófonos, aprendientes de español en el nivel B1, universitarios de entre 19 y 22 años.

En el proceso de validación de los datos de la muestra se eliminó a los informantes extranjeros o con un nivel de español fuera de rango. Esto es, perderíamos capacidad de comparación si los informantes no tuvieran un perfil lingüístico afín entre ellos.

La tableta utilizada fue un Ipad pro 10.5 pulgadas con un brillo fijo de la pantalla del $65 \%$. Grupo Experimental (tableta) Grupo de control (papel)

\begin{tabular}{lcc} 
Informantes & 42 & 26 \\
Lengua materna & Portugués & Portugués \\
Estudios & CSC & CSC \\
Edad & $19-20$ & $19-20$ \\
\hline
\end{tabular}

Tabla 1: Perfil de los informantes

\subsection{El texto}

El texto utilizado fue el siguiente:

«Yo nací en Lisboa pero vivo en Oporto. Cuando era niña, participé en muchos espectáculos de la escuela de baile. Me gusta mucho recordar esos momentos porque me lo pasaba muy bien e íbamos siempre de excursión. Además, salíamos de la rutina.

Uno de los regalos más especiales que recibí fue mi primer móvil. Lo recibí en un cumpleaños. Fueron mis padres quienes me lo regalaron y me dieron la sorpresa. Nunca lo olvidaré porque al día siguiente lo partí...

Hay un momento en mi vida del que tampoco me voy a olvidar. Cuando era más joven, participé en un concurso para conocer a mi actriz portuguesa favorita. Simplemente tenía que enviar un mensaje de texto y, entre millones de participantes, lo gané. Estuvimos un día paseando en Lisboa, conocí a su novio y después aparecimos en televisión. Me encantó estar con ella.»

En ambos textos se utilizó la tipografía Times New Roman de tamaño 12.

Como hemos referido el texto utilizado para esta comparación fue diseñado para propiciar las falsas predicciones y poner contar con más elementos de comparación que validaran los datos. Sabemos por otros trabajos (Chenoll 2013; 2016a) que el proceso de construcción en hablantes de lenguas afines es habitual debido a las semejanzas y a los procesos de ceguera al cambio que suceden en este proceso analítico. De ahí que se suponga que el proceso también se repetirá en una lectura de texto a pesar de existir datos inequívocos sobre la producción como es la letra escrita.

A modo de ejemplo, palabras como nací, móvil, dieron, conocer, a mi actriz o simplemente poseen un alto grado de potencial ceguera por falta de atención (Simons 
2000a; 2000b; Simons y Chabris 1999) con las estructuras nativas del portugués como nasci, móvel, dieran*/deram, conhecer ([kúneserer]) o la elisión de la preposición correspondiente al complemento directo de persona en conocer mi actriz*.

Como es evidente, en la contabilidad de los errores o transformaciones se obvió la clasificación de la pronunciación ya que el motivo podría ser un error de pronunciación y no de construcción debido al contexto en el que se lee. Esto es, si un informante lee [i íbamos] en el texto e íbamos, se considera un error de pronunciación y no de construcción.

Una vez realizada la prueba se pasó a su análisis de manera aislada de las 143 palabras que existen en el texto y escogiendo el tipo de errores derivados de la construcción del texto.

\subsection{Clasificación de los errores}

Para facilitar su análisis se seleccionaron 4 tipos de construcciones.

\section{a) Lengua materna}

Errores derivados directamente de su lengua materna. Ejemplos de estos son producciones como deram, gañé o recebí. Este tipo de error define el objetivo final de la prueba ya que la mente predictiva hace que los informantes transformen el texto real con el texto que es esperable según su interlengua. Este tipo de errores es directamente proporcional al estadio de la interlengua alcanzado (Chenoll 2016b).

\section{b) Creación pura}

Se refiere a errores que el informante comete independientemente de su lengua materna. Por ejemplo momiento. Si bien, la palabra en cuestión no forma parte de su lengua materna, sí que forma parte de su interlengua a través de un error de generalización en el que se establece una regla de diptongación en la $e$ tónica.

\section{c) Eliminación}

La lectura elimina o ignora ciertas partes que no son aceptables en su interlengua. Por ejemplo, la eliminación de la preposición $a$ del complemento directo de persona.

\section{d) Reformulación}

Durante la realización de la prueba se observó que algunos informantes leían incorrectamente la estructura pero que inmediatamente reformulaban el texto y lo leían de manera adecuada. A modo de ejemplo, si un informante leía móvel inmediatamente se daba cuenta del error y reformulaba su expresión a móvil.

\section{Resultados de la prueba}

A través del análisis posterior de los errores y su contabilización, pudimos observar que los errores se distribuyen de la siguiente manera en relación con el contexto de lectura.

Grupo Experimental (tableta) Grupo de control (papel) 


\begin{tabular}{lcc} 
Creación Pura & $26,66 \%$ & $23,88 \%$ \\
Eliminación & $5,56 \%$ & $6,1 \%$ \\
Reformulación & $18,89 \%$ & $13,8 \%$ \\
\hline
\end{tabular}

Tabla 2. Errores por informante en relación al contexto de la lectura

\subsection{Media de errores reales}

Desde el punto de vista del porcentaje de errores por texto e informante se pudo observar que el índice de errores más alto se da en los textos que fueron leídos en formato digital.

Grupo Experimental (tableta) Grupo de control (papel)

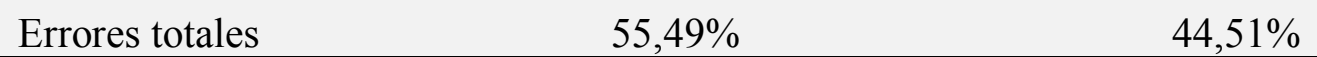

Tabla 3. Errores discriminados por contexto

Esto nos indica que, de manera general, existen más errores de lectura en un formato digital que en un formato analógico o de papel. De hecho, podemos observar que la diferencia porcentual es de un 10,98\% lo que puede suponer un problema en un aprendizaje en el que la observación de la diferencia marca el nivel de optimización como en el caso del aprendizaje de lengua afines.

\subsection{Errores con más ocurrencias}

Por su parte, los textos tuvieron una media de errores de 3 (en la lectura analógica) y de 5 en la lectura digital lo que refuerza la idea de que existe más errores en la pantalla que en el papel.

En este sentido, las mayores ocurrencias de errores surgieron en las palabras recibi por recebi; siguiente por seguinte*; gané por gañé*; simplemente por simplesmente*; un (mensaje) por una* (mensaje); conocer por coñecer* y después por depues*. Como se puede observar, todas ellas provienen de una gran influencia de la lengua materna del informante que predice y construye lo que está leyendo. De hecho, en varias ocasiones se llega incluso a añadir una $a$ al prever que la palabra mensaje es femenina como sucede en su lengua materna.

\section{Discusión de los resultados}

Como hemos podido observar, en todos los planos generales la lectura de un texto en un contexto analógico obtiene mejores resultados que en un formato digital.

Llama la atención, no obstante, que la influencia de la lengua materna se exprese con más fuerza (un 7,33\% más) en un contexto analógico que en el digital. Sin embargo, existe un proceso de reformulación mayor en un contexto digital que en el analógico (un 5,09\%).

Como parece evidente, las diferencias porcentuales no implican un cambio verdaderamente significativo en la construcción de la realidad lingüística. Puede, sí, existir una apreciación más efectiva en el texto analógico, pero no parece tener una relación directa y clara con la efectividad del proceso de lectura. Las características de un 
texto digital aislado (sin otras distracciones como los hipervínculos o las llamadas de atención de otros programas) como es el caso de la prueba expuesta, pueden llegar a perjudicar el proceso de lectura discreta, esto es, la discriminación de las diferencias en las cuales parece tener más peso el carácter predictivo influenciado por la interlengua del alumno que el propio contexto en el que se realiza la lectura.

Es relevante, a su vez, que el proceso de reformulación sea mayor en el texto digital lo que puede implicar que, si bien en un primer momento pueda perjudicar el proceso de lectura, es rápidamente subsanado por la normal idiosincrasia de la lectura sincrética en el que el movimiento ocular realiza acciones de barrido rápido y corrige, si es oportuno, la mala interpretación primigenia. Esto es, existe una minimización del error que se activa de manera más funcional en el texto digital.

\section{Conclusiones}

En definitiva, creemos probado que existe un alto grado de construcción lectora en ambos contextos incluso en porcentajes similares con ciertas tendencias dependiendo del proceso que esté en análisis.

De la misma manera, creemos que, a pesar de observar un menor rendimiento en el proceso de discriminación y predicción en el contexto digital, esto no es impedimento para continuar trabajando en este contexto. No solo porque la diferencia no es significativa desde el punto de vista general, sino porque las ventajas del contexto digital son innumerables en lo que se refiere al proceso de enseñanza y aprendizaje de una lengua extranjera.

Tendiendo en cuenta estos resultados, podríamos señalar que es preferible, en un primer estadio de desarrollo de la interlengua, tender a usar formatos que ayuden a la discriminación de los elementos diferenciadores a través de textos analógicos $\mathrm{y}$, progresivamente sustituir este contexto por otros más ricos como los que ofrece el formato digital.

Finalmente, creemos fundamental considerar la discriminación de elementos semejantes como una herramienta metacognitiva que ayude al alumno de lenguas extranjeras y particularmente de lenguas afines o próximas a la optimización del proceso de enseñanza y aprendizaje, sea este más o menos digitalizado.

\section{Bibliografía}

BRAVO VALDIVIESO, L. (2016). «El aprendizaje del lenguaje escrito y las ciencias de la lectura. Un límite entre la psicología cognitiva, las neurociencias y la educación.» Limite. Revista Interdisciplinaria fe Filosofia y Psicología, 11(36), 50-59.

ChenOll MorA, A. (2013). «El desarrollo de la competencia oral en internet: procesos, recursos y aplicaciones en el aula.» Actas del V Congreso sobre la Enseñanza del Español en Portugal, Aveiro. pp. 202-215. Recuperado el 30/07/18, de http://www.mecd.gob.es/portugal/dms/consejeriasexteriores/portugal/publicaciones/ActasVcongresoELEPortugal.pdf 
Chenoll Mora, A. (2016a). «Ahora me ves: la percepción como principio básico del aprendizaje de lenguas extranjeras», Foro de Profesores ELE 12(1), 21-31. Recuperado el 30/07/18, de https://ojs.uv.es/index.php/foroele/article/view/9164/8682

Chenoll MorA, A. (2016b). «El español invisible: Una aproximación a la ilusión de la percepción fonética y escrita del español en portugueses y su aplicación al proceso de aprendizaje y evaluación.» El Español como Lengua Extranjera en Portugal: Retos De La Enseñanza De Lenguas Cercanas, (II), 26-35. Recuperado el 30/07/18, de https://www.mecd.gob.es/portugal/dms/consejeriasexteriores/portugal/publicaciones/retos_2016_final2/retos_2016_final3.pdf

CORDER, S. P. (1967). «The significance of learners' errors». IRAL, 5, 161-170, recogido en Corder, S. P. (1981). Error Analysis and Interlanguage. Oxford: Oxford University Press.

CORDER, S. P. (1971). «Idyosincratic dialects and error analysis». International Review of Applied Linguistics 9. 149-159

FERNÁNDEZ, S. (1997). Interlengua y análisis de errores en el aprendizaje del español como lengua extranjera. Edelsa. Madrid.

Hohwy, J. (2013). The Predictive Mind. Oxford University Press. Oxford.

McMillan, J. J., Y ReVIEW, R. W. A. (1993). «Auditors' Belief Revisions and Evidence Search: The Effect of Hypothesis Frame, Confirmation Bias, and Professional Skepticism.» Jstor, 443-465.

NEMSER, W. (1971). «Approximative systems of foreign language learners». En IRAL IX (2), 115-123.

NEMSER, W. (1992). «Los sistemas aproximados de los que aprenden lenguas segundas.» en J. Muñoz Liceras (Ed.), La adquisición de las lenguas extranjeras (pp. 51-61). Madrid: Visor.

PIQUER, I. (2016). «La adquisición del proceso lector en lengua extranjera.» REICE. revista iberoamericana sobre calidad, eficacia y cambio en educación, 14(1), 37-47.

PORQUIER, R. (1975). Analyse d'erreurs en français langue étrangère: études sur les erreurs grammaticales dans la production orale libre chez les adultes anglophones. Universidad de París, VIII.

RANSOM, M., FAZElPOUR, S., Y Mole, C. (2017). «Attention in the predictive mind.» Consciousness and Cognition, 47, 99-112.

SimONS, D. J. (2000A). «Attentional capture and inattentional blindness.» Trends in Cognitive Sciences, 4(4), 147-155.

Simons, D. J. (2000b). Change blindness and visual memory. Hove, East Sussex: Psychology Press.

SiMONS, D. J., Y CHABRIS, C. F. (1999). «Gorillas in our midst: Sustained inattentional blindness for dynamic events.» Perception, 28(9), 1059-1074.

TARONE, E. (1994). “Interlanguage”, en Elsevier Ltd., 4, pp. 1715-1719. Recuperado el 30/07/18, de http://socling.genlingnw.ru/files/ya/interlanguage\%20Tarone.PDF 